\title{
Relations between Facial Display, Eye Gaze and Head Tilt: Dominance Perception Variations of Virtual Agents
}

\author{
Nikolaus Bee, Stefan Franke and Elisabeth André \\ Institute of Computer Science, Augsburg University, \\ 86135 Augsburg, Germany \\ \{bee, andre\}einformatik.uni-augsburg.de
}

\begin{abstract}
In this paper, we focus on facial displays, eye gaze and head tilts to express social dominance. In particular, we are interested in the interaction of different non-verbal cues. We present a study which systematically varies eye gaze and head tilts for five basic emotions and a neutral state using our own graphics and animation engine. The resulting images are then presented to a large number of subjects via a web-based interface who are asked to attribute dominance values to the character shown in the images. First, we analyze how dominance ratings are influenced by the conveyed emotional facial expression. Further, we investigate how gaze direction and head pose influence dominance perception depending on the displayed emotional state.
\end{abstract}

\section{Introduction}

In order to come across as believable, virtual agents need to portray social behaviors in a convincing manner. Among other things, social behaviors are reflected by the way a character communicates social dominance. Prior psychological experiments indicate that the following facial cues are used to express social dominance: facial expressions, eye gaze and head tilts.

There is empirical evidence that the facial display of emotions influences the perception of dominance. Knutson [7] conducted an empirical study in order to investigate how the facial display of the five basic emotions and a neutral expression influences the assessment of dominance. He observed that happy, angry and disgusted faces received higher values of dominance than fearful, sad and neutral faces. Mignault and Chaudhuri [11] found that a bowed head is perceived as submissive while a raised head expresses dominance.

Further studies investigate if there is an interaction between perceived gaze direction and perceived facial expression. Adams and Kleck [1] observed that direct gaze supports the perception of approach-oriented emotions (such as anger and joy) while averted gaze enhances the perception of avoidance-directed emotions (such as fear and sadness). Sander and colleagues [16] found that the emotions fear and anger were perceived with significantly different intensity dependent on averted or direct gaze. Fear was rated more intense in combination with averted gaze whereas anger was rated more 
intense in combination with direct gaze. They did not map, however, the ratings onto the dimensions of an emotional model, such as the pleasure, arousal and dominance dimensions of the PAD model [10].

Lance and Marsella [8] developed a gaze model for emotional expression. They used the PAD model and a simple list of emotions to evaluate their gaze model with varying parameters. They found that a virtual character with either a raised head or a bowed body and/or fast movements appears more dominant. Low dominance was found for a bowed head and/or a neutral body posture without fast movements.

Grammer and Oberzaucher [5] mapped the facial expressions of a virtual character onto Russell's Circumplex Model [13], which is a two dimensional emotion model with the dimensions arousal and pleasure. They conducted a study with 409 subjects, varied the action units of a FACS-enabled female virtual face and analyzed how the activation of single action units relate to the Circumplex Model. To map the action units onto the arousal-pleasure space of Russell's model, they asked the subjects to rate 18 pairs of words recommended by Mehrabian and Russell [10].

In this paper, we will investigate how to orchestrate facial expressions, eye gaze and head tilts when a virtual agent is expected to express social dominance. We use a virtual character whose facial expression is controllable through FACS (Facial Action Coding System) [3]. To analyze social dominance, we apply methods from Mehrabian and Russell [9] [10], who defined the PAD (Pleasure, Arousal and Dominance) model for emotions by using bipolar pairs of words to judge humans' affective attitudes towards different situations. Based on the PAD model, Russell and Mehrabian created a dictionary with 151 emotional terms mapped onto pleasure, arousal and dominance [14].

In the following, we will present an experiment we conducted in order to investigate how facial expressions, gaze and head tilts of a virtual character influence the perception of dominance. On the basis of related studies, we expected the following outcomes:

- Dominance perception of a virtual human is influenced by the facial display of an emotion. In particular, facial expressions that convey joy, anger and disgust are perceived as more dominant than neutral, fearful or sad facial expressions.

- The perception of dominance depends on the direction of the eye gaze and head orientation. In particular, people who avert their gaze and look down appear to be more submissive than people using direct gaze and looking up.

- There are interactions between emotional facial displays, head and eye gaze direction. In particular, we assume that direct eye gaze in combination with upward head orientation will increase dominance ratings for joy, anger and disgust while averted gaze in combination with downward head orientation will decrease them. Vice versa, we expect that averted eye gaze in combination with downward head orientation will decrease the dominance values for fear and sadness while direct eye gaze in combination with upward head orientation will increase them.

\section{Method}

\subsection{Affective and Attentive Virtual Character}

For our study, we used a fully controllable virtual head which was developed by Augsburg University and which is freely available [6]. The head is based on the Facial 
Action Coding System (FACS) developed by Ekman and Friesen [3] to classify human facial expressions. It divides the face into action units (AU) to describe the different expressions a face can display (e.g. inner brow raiser, nose wrinkler, or cheek puffer). Although FACS was originally designed to analyze natural facial expressions, it turned out to be usable as a standard for production purposes as well. That is why FACSbased coding systems are used for the generation of facial expressions displayed by virtual characters, such as Kong in Peter Jackson's King Kong [15]. But the usage of FACS is not limited to virtual characters in movies. The gaming industry with HalfLife 2 by Valve also utilizes the FACS system to produce the facial expressions of their characters [17].

Alfred (see Fig. 1), a butler-like character, uses these action units to synthesize a huge amount of different facial expressions. The action units are designed using morph targets and thus give a designer the full power in defining the facial expression outlook. The system includes a tool to control the single action units, which enables us to store the result in an XML file for later usage in our agent system [2].

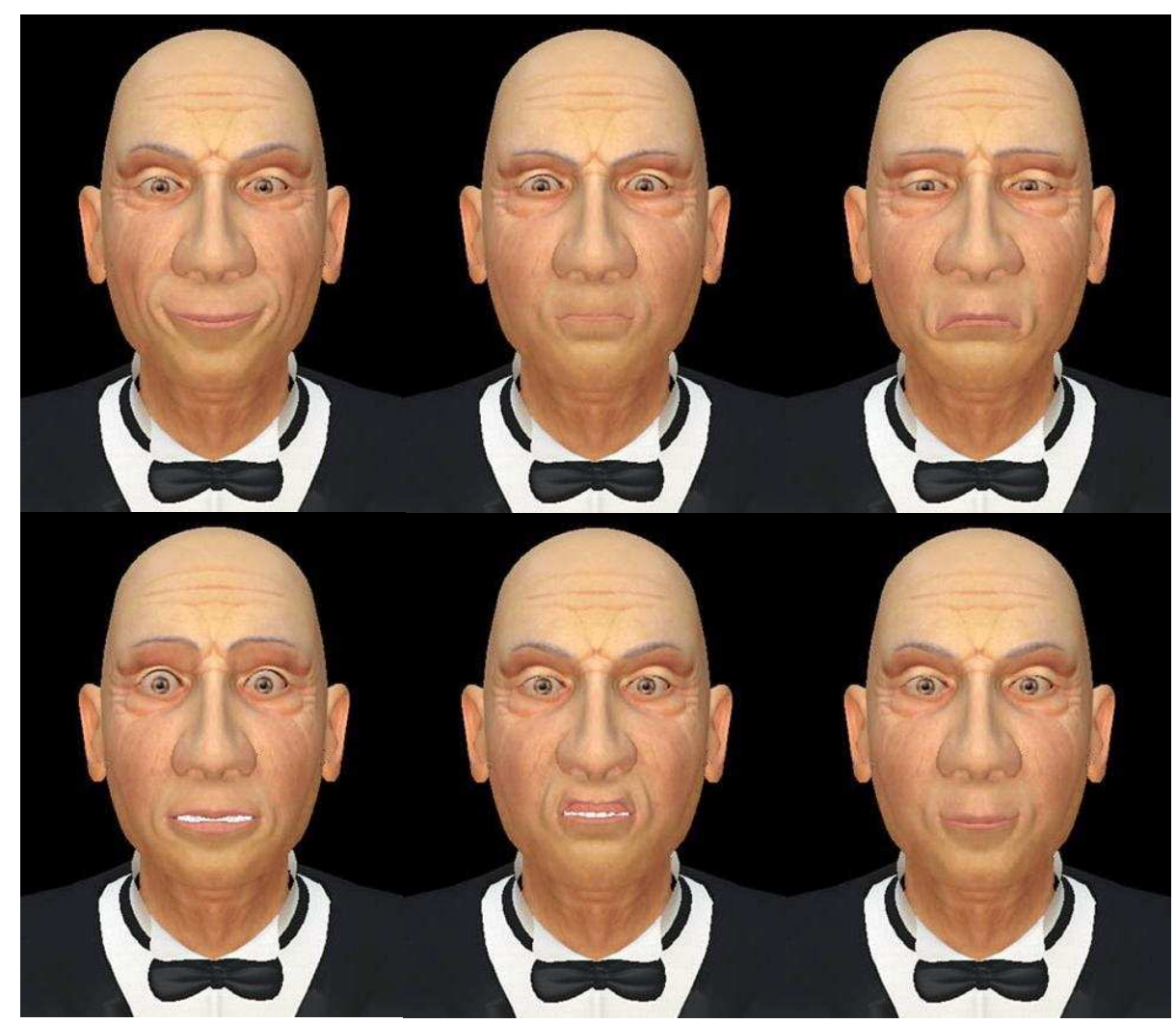

Figure 1: The six facial expressions of Alfred used in the study. (Upper row from left to right: joy, anger and sadness; lower row: fear, disgust and neutral)

Alfred's mesh has a resolution of about 21.000 triangles. For displaying more expressive wrinkles in the face, normal maps baked from a high-resolution mesh are used [12]. The morph targets for the action units are modeled following the actor's templates from the Facial Expression Repertoire (FER) [4].

The Horde3D GameEngine [6] provides an inverse kinematics component with 
which the head and eye direction can be controlled. The head and eye direction can be set independently by the IK component (e.g. the head can look to the right while the eyes are directed towards the center).

\subsection{Stimuli}

The parameters for the affective display of the virtual character were picked from the Facial Expression Repertoire (FER) [4]. The FER provides samples of over 150 facial expressions that may be mapped onto the action units of FACS. The database does not only indicate which action units have to be activated for certain facial expressions, it also provides a rich dataset of videos which show how the action units ought to be designed. We chose the following representatives: Joy (action units 6 and 12), Anger (action units 4, 5, 7, 17 and 23), Sadness (action unit 1, 4, 7, 11, 15 and 17), Fear (action units 1, 2, 4, 5, 20 and 25) and Disgust (action units 10 and 17).

We varied the attentiveness of the virtual character by modifying head orientation and eye gaze direction. The maximum angle for the eye gaze was defined by the limits of the pupils' visibility and also applied for the head orientation. This led to nine gaze orientations for the head and eyes (up (N), center $(\mathrm{C})$ and down $(\mathrm{S})$ varied by $\pm 8.0^{\circ}$ and left $(\mathrm{W})$, center $(\mathrm{C})$ and right $(\mathrm{E})$ varied by $\left.\pm 8.5^{\circ}\right)$.

The possible combinations of 6 emotions $\times 9$ head directions $\times 9$ eye directions result into 486 different facial displays. To reduce them to a reasonable amount, we assumed that it does not matter whether the virtual character gazes to the left or to the right and randomly distributed the horizontal gaze to one of these sides. To further limit the amount of facial expressions, we removed the facial expressions where the eye is gazing into the opposite direction of the head orientation (i.e. the head is directed to the left and the eyes are directed to the right). These two assumptions limit the number of possible facial displays to 194 . We automatically generated pictures from these settings with a script which controls Alfred and automatically saves the expression as a screenshot.

\subsection{Questionnaire}

To obtain the dominance values for the affective and attentive facial expressions, we followed the instructions from Mehrabian and Russell [10]. They provide 18 pairs of words, 6 for each of the dimensions (i.e. pleasure, arousal and dominance), which need to be rated on a 9-point scale. As we were mainly interested in the dominance factor of the facial displays, we limited these pairs of words to the six that are necessary for obtaining the dominance factor (i.e. Controlling - Controlled, Influential - Influenced, In control - Cared-for, Important - Awed, Dominant - Submissive and Autonomous Guided).

\subsection{Study}

69 (40 female and 29 male) participants judged in total 862 pictures. The mean age was 28.5 and the participants came from all walks of life. Each of the seven emotions was judged about 123 times and each of the 194 pictures was judged 4.4 times on average, whereby every picture was judged at least 4 times, but maximally 5 times. On average, the subjects rated their experience in 3D modeling with 0.49 , their experience in animating facial expressions with 0.23 and their background in emotion research 
with 0.36 on a scale between 0 and 4 (with 0 representing no experience at all). That is our subjects had no experience in any related field.

\section{Results}

The analysis of dominance ratings for the different combinations of eye gaze, head pose and emotional displays was based on the one-way analysis of variance (ANOVA) across the different groups and the Tukey-HSD for the post-hoc two-sided pairwise comparisons. $t$-Tests where applied two-sided, as we did not predefine, in which direction the means differed.

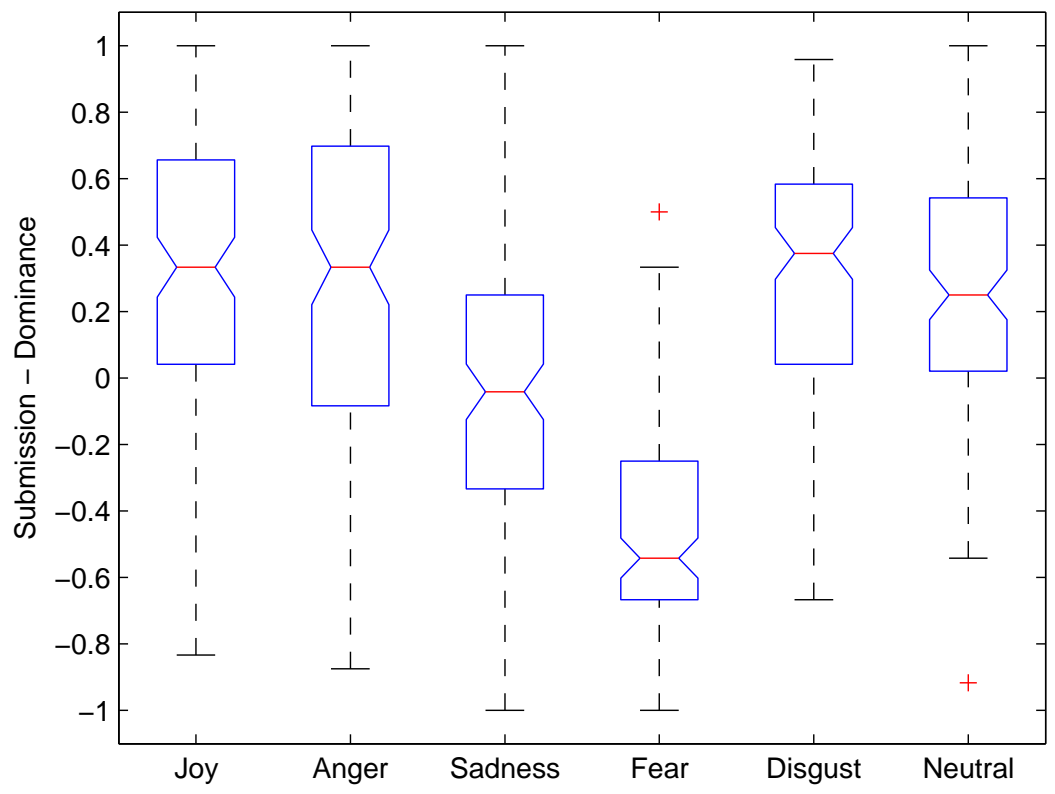

Figure 2: Dominance values with all eye and head orientation variations

\subsection{Influence of Emotional Displays, Gaze and Head Tilts on Dom- inance Perception}

\subsubsection{Influence of Emotional Displays on Dominance Perception.}

A comparison of all six facial expressions including all variants of the gaze and head orientations with ANOVA revealed significant differences in dominance perception among the emotion groups $\left(F(5,705)=74.6, p=0, \eta^{2}=11.9\right)$. The pairwise Tukey-HSD post-hoc analysis revealed significant differences between all groups, except Joy - Anger, Joy - Disgust, Joy - Neutral, Anger - Disgust, Anger - Neutral and Disgust-Neutral (see Fig. 2 and Tab. 2).

When comparing our results to Russel's and Mehrabian's [14] dominance values 


\begin{tabular}{r|cc}
\hline & $\begin{array}{c}\text { Russell \& } \\
\text { Mehrabian }\end{array}$ & $\begin{array}{c}\text { Our } \\
\text { Experiment }\end{array}$ \\
\hline Joy & 0.35 & 0.32 \\
Anger & 0.25 & 0.29 \\
Sadness & -0.33 & -0.04 \\
Fear & -0.43 & -0.46 \\
Disgust & 0.11 & 0.31 \\
\hline
\end{tabular}

Table 1: Comparison between Russell and Mehrabian's and our dominance values

for the emotions Joy, Anger, Sadness, Fear and Disgust, we noticed only small differences for Joy, Anger and Fear (see Tab. 1).

Furthermore, most of our results are in line with a study conducted by Knutson [7]. Joyful, angry or disgusted facial displays were rated more dominant than fearful and sad facial displays. As Knutson, we got the highest dominance value for joy and the lowest dominance value for fear. However, unlike Knutson, we observed that neutral facial expressions were perceived nearly as dominant as joyful or angry facial expressions.

\subsubsection{Influence of Gaze on Dominance Perception}

In the previous paragraph, the influence of eye gaze and head orientation on dominance ratings was not separately analyzed. In the following, we will investigate how the gaze influences the perception of dominance whereby we will compare the effect of directed with the effect of averted gaze.

Dominance of direct gaze The one-way ANOVA revealed significantly different dominance ratings within all six groups of affective displays $(F(5,19)=7.96, p=0$, $\left.\eta^{2}=1.04\right)$ when the eyes and the head were directed at the user. A post-hoc analysis showed significant differences between several emotions. The Tukey-HSD analysis revealed that Joy significantly differed from Anger $(p<0.01)$, Fear $(p<0.001)$ and Sadness $(p<0.05)$. And further, it revealed significant differences between Fear and Disgust $(p<0.01)$ and between Fear and Neutral $(p<0.05)$ (see Fig. 3).

Dominance of Averted Gaze The one-way ANOVA for averted eye/head gaze revealed significantly different dominance values within the groups of all six affective displays independent from the direction of the averted gaze $(F(5,680)=71, p=0$, $\eta^{2}=11.3$ ). A post-hoc analysis shows significant differences between several groups of emotions. Table 2 shows the results for the Tukey-HSD post-hoc analysis between the different emotion groups, where, for example, sadness or fear significantly differs from all the other emotion groups.

Direct vs. Averted Gaze A comparison of averted and direct eye/head gaze revealed significant differences for Joy and Anger. The dominance value of Joy dropped from 0.80 for directed gaze to 0.33 for averted gaze. A two-tailed $t$-test revealed that the dominance value for averted gaze was significantly lower $(t=-4.1, d f=4.1, p<$ 0.01 ) than the dominance value for direct gaze. In contrast the dominance value for Anger rose from -0.33 in the case of direct gaze to 0.38 in the case of averted gaze. 


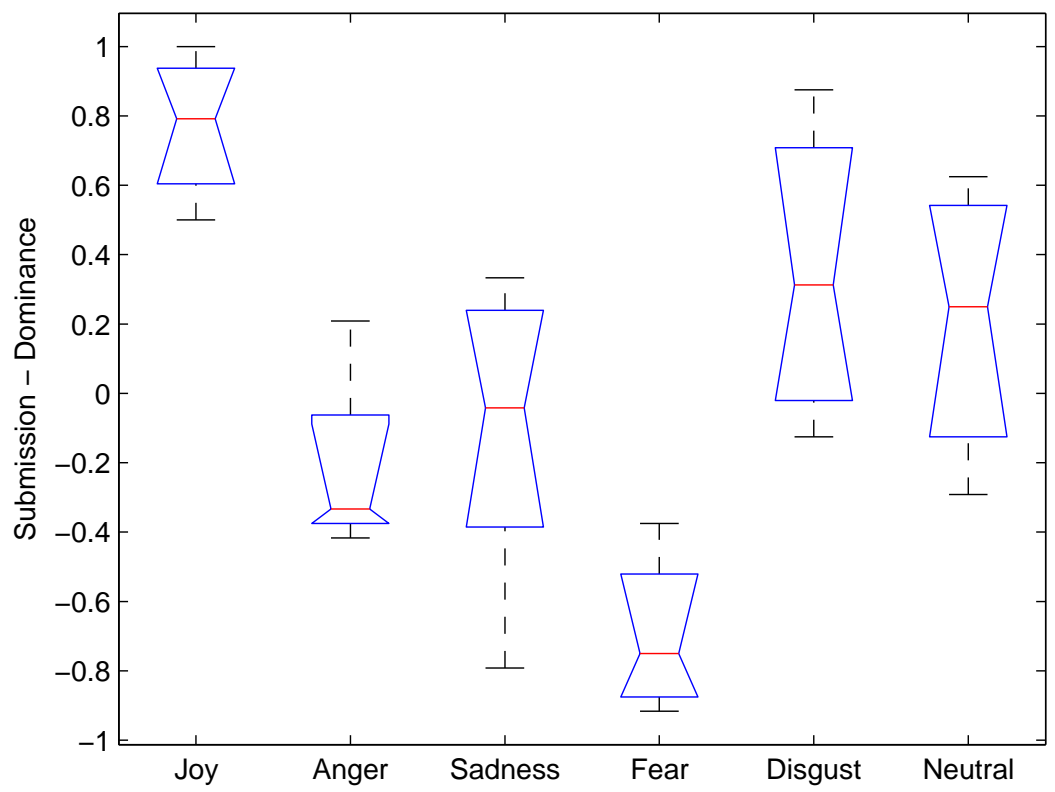

Figure 3: Dominance values with eyes and head directed at the user

A two-tailed $t$-test revealed that the dominance value of averted gaze was significantly higher $(t=3.6, d f=4.1, p<0.05)$ than that of direct gaze. Fear $(p=0.1)$, Sadness $(p=0.57)$, Disgust $(p=0.9)$ and the Neutral $(p=0.86)$ affective display were independent from the virtual character's current eye/head gaze (see Tab. 3). They did not show any significant differences for averted versus directed eye/head gaze.

\subsection{Influence of Eye Gaze in Combination with Facial Displays on Dominance Perception}

As head orientation dominates eye gaze, we keep the head oriented towards the user and limit our analysis here to different eye gaze directions.

In Section 3.1.2, significant differences between averted and directed gaze were

\begin{tabular}{r|cccccc}
\hline & Joy & Anger & Sadness & Fear & Disgust & Neutral \\
\hline Joy & - & n.s. & $* * *$ & $* * *$ & n.s. & n.s. \\
Anger & n.s. & - & $* * *$ & $* * *$ & n.s. & n.s. \\
Sadness & $* * *$ & $* * *$ & - & $* * *$ & $* * *$ & $* * *$ \\
Fear & $* * *$ & $* * *$ & $* * *$ & - & $* * *$ & $* * *$ \\
Disgust & n.s. & n.s. & $* * *$ & $* * *$ & - & n.s. \\
Neutral & n.s. & n.s. & $* * *$ & $* * *$ & n.s. & - \\
\hline
\end{tabular}

Table 2: Post-hoc comparisons for averted eye gaze and head orientation between different emotions $\left({ }^{* * *} p=0\right.$, n.s. $=$ not significant $)$ 


\begin{tabular}{r|rr}
\hline & Direct & Averted \\
\hline Joy & 0.80 & 0.33 \\
Anger & -0.33 & 0.38 \\
Sadness & -0.04 & -0.04 \\
Fear & -0.75 & -0.52 \\
Disgust & 0.31 & 0.38 \\
Neutral & 0.25 & 0.25 \\
\hline
\end{tabular}

Table 3: Median values for dominance over all emotions with directed eye and head direction compared with averted eye and head direction

observed for Anger, Fear and Joy. When we varied the direction of eye gaze with the head oriented directly towards the user, the one-way ANOVA revealed significant differences in dominance perception for Anger $\left(F(5,20)=4.2, p<0.001, \eta^{2}=0.49\right)$. The post-hoc Tukey-HSD revealed only significant differences between the following eye gaze directions: North - Center $(p<0.05)$ and North - East $(p<0.05)$ (see Fig. 4). For Joy or Fear, we did not observe any significant differences in dominance perception for the chosen eye gaze directions.

Direct vs. Averted Eye Gaze A two-tailed $t$-test on the dominance values assigned to Joy for direct and averted eye gaze while the head gaze was oriented towards the user revealed that the dominance value for averted gaze $(\mathrm{D}=0.35)$ was significantly higher $(t=-3.0, d f=7.4, p<0.05)$ than the dominance value for direct gaze $(\mathrm{D}=0.77)$. In contrast, the dominance value for Anger rose from -0.22 to 0.17 . However, this difference was not significant $(t=2.2, d f=5.9, p=0.07)$. Fear, in comparison to Section 3.1.2 (Direct vs. Averted Gaze), also did not show a significant difference between direct and averted eye gaze, when the head orientation was directed towards the user $(t=1.6, d f=5.0, p=0.17)$.

\subsection{Influence of Head Orientation in Combination with Facial Dis- plays on Dominance Ratings}

Significant effects of different head orientations on dominance ratings were only obtained for Anger $\left(F(5,113)=2.9, p<0.05, \eta^{2}=0.65\right)$, Sadness $(F(5,114)=2.5$, $\left.p<0.05, \eta^{2}=0.44\right)$ and Neutral $\left(F(5,114)=2.7, p<0.05, \eta^{2}=0.33\right)$ using a oneway ANOVA. A pairwise comparison for Anger within the groups with the post-hoc Tukey-HSD analysis revealed only significant differences between Center and North East ( $p<0.05$ ) head orientation (see Fig. 5).

For Sadness, a post-hoc analysis did not reveal any significant differences with pairwise group comparisons. For Neutral, a Tukey-HSD post-hoc analysis showed significant group differences for North - South $(p<0.05)$ and North - West $(p<0.05)$ head orientation (see Fig. 6).

Raised vs. Lowered Head For the six displayed emotions, we analyzed how a raised $(N W, N$ and $N W)$ in comparison to a lowered $(S W, S$ and $S E)$ head influenced the perception of dominance. A two-tailed $t$-test on a Neutral facial expression revealed 


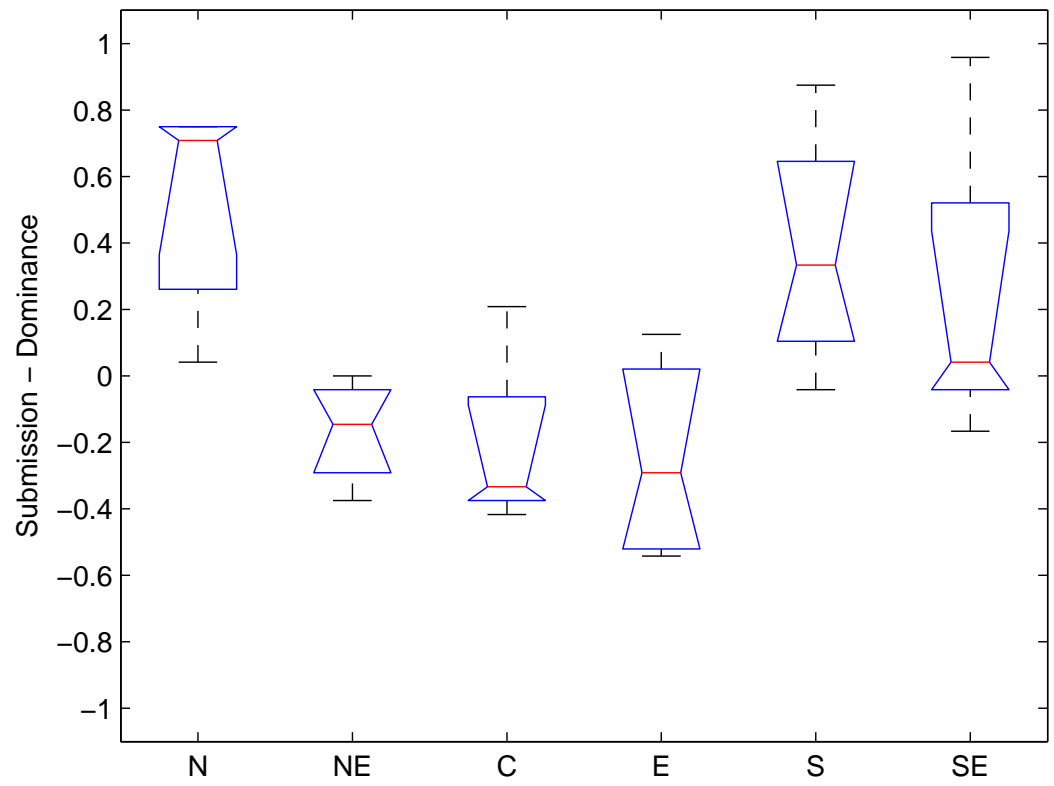

Figure 4: Dominance values for anger with directed head orientation and different eye gaze directions

significant differences between raised $(\mathrm{D}=0.41)$ and lowered $(\mathrm{D}=0.20)$ head orientations $(t=2.30, d f=66.1, p<0.01)$. These findings are in line with results from Lance and Marsella [8], who analyzed perceived dominance for a virtual character without facial expressions.

In addition to Lance and Marsella, we found significant effects for angry and sad facial displays. In the case of Anger, the dominance value varied from 0.52 for a raised head to 0.22 for a lowered head $(t=2.57, d f=64.0, p<0.05)$; in the case of Sadness it varied from 0.15 for a raised head to -0.16 for lowered head $(t=3.26$, $d f=58.3, p<0.01)$. For the other investigated emotions, we did not find any significant differences between a bowed and a raised head.

That is, in the case of Anger, Sadness and Neutral, a raised head led to a higher dominance value compared to a lowered head.

Raised Head and Direct Eye Gaze vs. Lowered Head and Averted Eye Gaze We also investigated whether the effect obtained could be increased by showing a raised head in combination with direct eye gaze and a lowered head in combination with averted eye gaze. A significant difference in dominance ratings was observed for Anger and Disgust. A two-tailed $t$-test showed that the dominance value for Anger rose from 0.26 for a lowered head in combination with averted eye gaze to 0.76 for a raised head in combination with direct eye gaze $(t=4.0, d f=29.0, p<0.001)$. Also the dominance value for Disgust rose significantly from 0.27 in the case of a raised head and direct eye gaze to 0.54 in the case of a lowered head and averted eye gaze $(t=2.5$, $d f=31.8, p<0.05)$. Joy could not be found to differ significantly $(p=0.34)$, just 


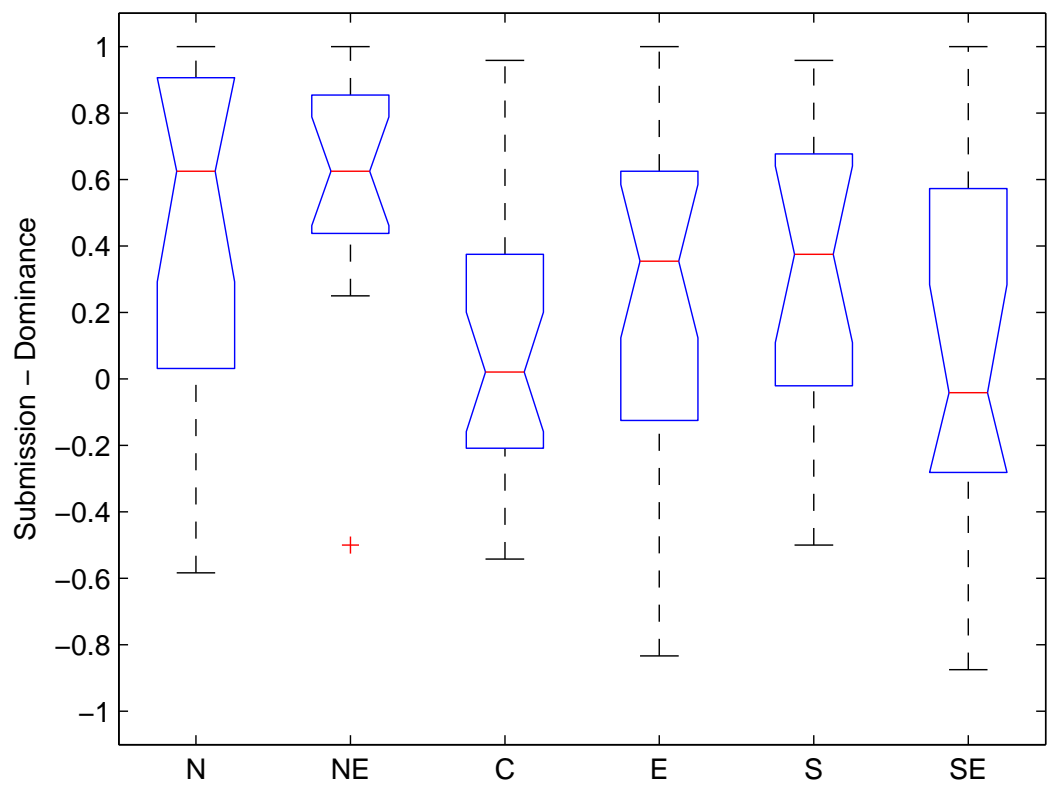

Figure 5: Dominance values for anger and head orientation

the same as Fear $(p=0.38)$ and Sadness $(p=0.24)$.

\subsection{Perception Test}

To check whether the chosen stimuli were perceived as they were meant, we conducted a small-scale study. We recruited eight subjects and presented them with screenshots of our virtual character (see Fig. 1) showing the six emotions with the head and the eyes directed towards the subjects. The subjects had to decide which emotion word out of a list with six emotion words (joy, anger, sadness, fear, disgust and neutral) matched the currently shown facial expression best. Further, they were informed that one and the same emotion could be assigned to several faces and that they were allowed to name more than one emotion in case they had the impression that several emotions could fit.

\begin{tabular}{r|cccccc}
\hline & Joy & Anger & Sadness & Fear & Disgust & Neutral \\
\hline Joy & 8 & - & - & - & - & - \\
Anger & - & 5 & 1 & - & 3 & 1 \\
Sadness & - & - & 8 & - & - & - \\
Fear & - & - & - & 8 & - & - \\
Disgust & - & 4 & - & - & 7 & - \\
Neutral & 4 & - & - & - & - & 7 \\
\hline
\end{tabular}

Table 4: Results of the perception test for emotional expressions of Alfred. Multiple nominations were allowed.

Table 4 presents the results of this test. The rows indicate onto which emotions the 


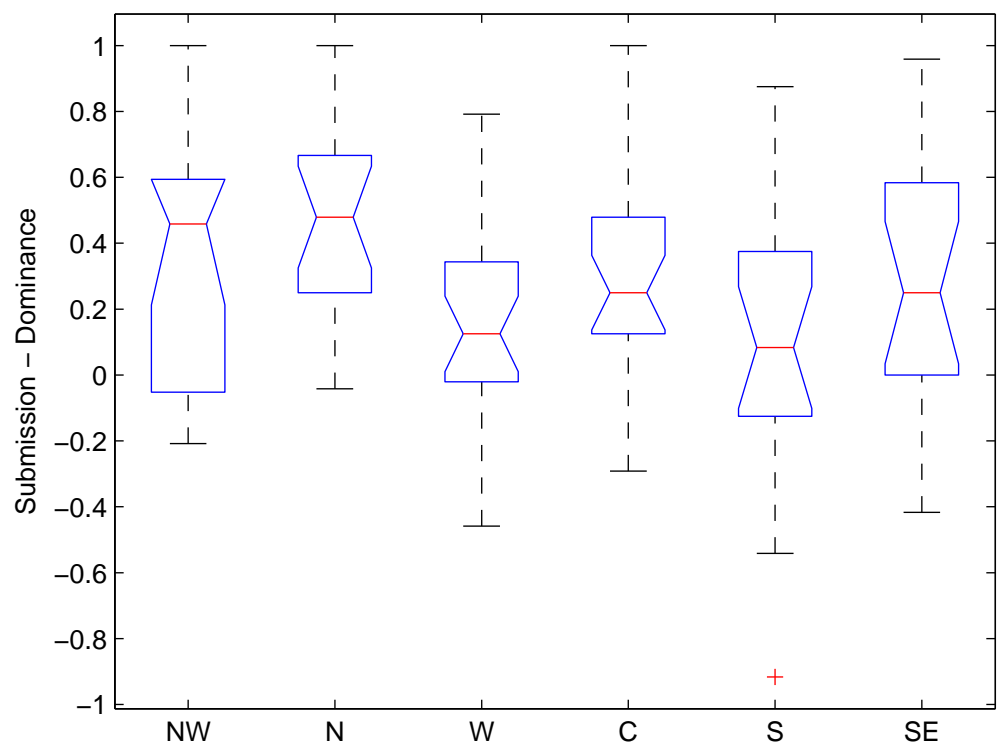

Figure 6: Dominance values for neutral and head orientation

users mapped the shown facial expressions. For example, the display for disgust was recognized three times as anger and six times as disgust. To summarize the results, the displays for joy, sadness and fear were in all cases perceived as the emotion derived from the Facial Expression Repertoire [4]. Anger and disgust were a bit overlapping with each other. Neutral was perceived four times as joy, which is understandable when looking at Alfred's neutral face (see Fig. 1), which seems to be slightly smiling. Overall, the subjects were more or less able to assign the intended emotions onto the displayed facial expressions. As a consequence, we could exclude side effects resulting from badly displayed emotions.

\section{Conclusion}

In this paper we investigated how the interplay of facial display, eye gaze and head tilts determines the perception of dominance. We could show that dominance ratings are influenced by emotional facial expressions. Higher dominance values were found for facial expressions conveying joy, anger and disgust. The dominance rating for a neutral facial expression, however, was significantly lower than that for joy, anger or disgust. Sadness and fear were perceived significantly less dominant in our experiment than joy, anger, disgust and neutral.

Further, we analyzed how dominance perception changes with varying eye gaze direction and head orientation. Our hypothesis that an averted gaze increases the degree of perceived submissiveness could not be confirmed in general. Only joy was perceived as less dominant when the gaze was averted. In contrast, anger and fear led to an increase in dominance in combination with averted gaze. Further, we found that gaze aversion had no influence on dominance ratings in combination with faces showing 
sadness, disgust or a neutral expression. Significant differences between an upward and downward directed head orientation could only be found for a neutral state, anger and sadness. Here, a lowered head orientation reduced the perception of dominance.

Finally, we could show that an upward head orientation in combination with direct eye gaze was rated as significantly more dominant than a downward oriented head with averted eye gaze direction for anger and disgust.

To summarize our findings, it matters where a virtual agent directs its attention dependent on its current affective state, and such effects need to be taken into account when modelling attentive affective agents.

\section{Acknowledgements}

This work has been funded in part by the European Commission under grant agreement DynaLearn (FP7-ICT-231526).

\section{References}

[1] R. B. Adams and R. E. Kleck. Effects of direct and averted gaze on the perception of facially communicated emotion. Emotion, 5(1):3-11, March 2005.

[2] N. Bee, B. Falk, and E. André. Simplified facial animation control utilizing novel input devices: A comparative study. In International Conference on Intelligent User Interfaces (IUI '09), pages 197-206, 2009.

[3] P. Ekman and W. Friesen. Unmasking the Face. Prentice Hall, 1975.

[4] Facial Expression Repertoire. Filmakademie Baden-Württemberg. http:// research.animationsinstitut.de/.

[5] K. Grammer and E. Oberzaucher. The reconstruction of facial expressions in embodied systems: New approaches to an old problem. In ZiF Mitteilungen, volume 2, pages 14-31, 2006.

[6] Horde3D GameEngine. University of Augsburg. http://mm-werkstatt. informatik.uni-augsburg.de/projects/GameEngine/.

[7] B. Knutson. Facial expressions of emotion influence interpersonal trait inferences. Journal of Nonverbal Behavior, 20(3):165-182, September 1996.

[8] B. Lance and S. Marsella. The relation between gaze behavior and the attribution of emotion: An empirical study. In Intelligent Virtual Agents (IVA '08), pages 1-14, 2008.

[9] A. Mehrabian. Framework for a comprehensive description and measurement of emotional states. Genetic, social, and general psychology monographs, 121(3):339-361, August 1995.

[10] A. Mehrabian and J. A. Russell. An Approach to Environmental Psychology. The MIT Press, 1974.

[11] A. Mignault and A. Chaudhuri. The many faces of a neutral face: Head tilt and perception of dominance and emotion. Journal of Nonverbal Behavior, 27(2):111-132, June 2003.

[12] C. Oat. Animated wrinkle maps. In SIGGRAPH '07: ACM SIGGRAPH 2007 courses, pages 33-37, New York, NY, USA, 2007. ACM.

[13] J. A. Russell. A circumplex model of affect. Journal of Personality and Social Psychology, 39:1161-1178, 1980.

[14] J. A. Russell and A. Mehrabian. Evidence for a three-factor theory of emotions. Journal of Research in Personality, 11(3):273-294, September 1977.

[15] M. Sagar. Facial performance capture and expressive translation for king kong. In SIGGRAPH '06: ACM SIGGRAPH 2006 Sketches, page 26, New York, NY, USA, 2006. ACM. 
[16] D. Sander, D. Grandjean, S. Kaiser, T. Wehrle, and K. R. Scherer. Interaction effects of perceived gaze direction and dynamic facial expression: Evidence for appraisal theories of emotion. European Journal of Cognitive Psychology, 19(3):470-480, 2007.

[17] Valve. Facial Expressions Primer from Half-Life 2 by Valve. http://developer. valvesoftware.com/wiki/Facial_Expressions_Primer. 